\title{
Vida útil de tapas de empanadas con películas biodegradables como separadores
}

\section{Shelf life of pie caps with biodegradable films as spacers}

\author{
Escobar Gianni, Daniela Verónica ${ }^{(1)}$, Sala, Angelina ${ }^{(2)}$, Silvera, Carlos ${ }^{(2)}$, Harispe, Rodrigo ${ }^{(3)}$, Márquez Romero, Rosa ${ }^{(1)}$ \\ (1) Gerencia de Proyectos Alimentarios, Laboratorio Tecnológico del Uruguay, LATU, Uruguay - (2) Universidad Católica \\ del Uruguay Dámaso Antonio Larrañaga, UCU, Uruguay - ${ }^{(3)}$ Alimentos RH, Uruguay \\ Contacto: descobar@latu.org.uy \\ Recibido: 15/6/2012 - Aprobado: 28/9/2012
}

\begin{abstract}
Resumen
Las tapas de empanadas utilizan películas de polietileno como separadores. Este trabajo estudia la sustitución de los separadores convencionales por películas comestibles en base a aislado de proteína de suero (WPI) con sorbato de potasio como conservador. La finalidad es reemplazar una película convencional por una biodegradable, que no se pega en la masa y facilita la separación, buscando también un aumento de vida útil. Las películas elaboradas por el método de moldeo por compresión se utilizaron como separadores en tapas de empanadas sin sorbato de potasio en la masa (A) y con sorbato de potasio en la masa (B), y se compararon con los separadores convencionales de polietileno (C). Mensualmente y durante cuatro meses se realizó el análisis sensorial, microbiológico y de humedad de las tapas, así como de humedad y solubilidad en las películas. Ninguna de las muestras presentó deterioro microbiológico ni sensorial en los cuatro meses estudiados; los atributos presentaron ninguna o ligera diferencia en el tiempo de estudio. Entre muestras, las diferencias fueron ligeras: las mejores puntuaciones las tuvieron en color la muestra $\mathrm{A}$, en sabor la muestra $\mathrm{C}$ y en textura y agrado general las muestras B y C. Las películas comestibles tienen un interesante potencial para esta aplicación, aunque se debe trabajar en enmascarar el flavor del suero.

Palabras clave: Películas biodegradables, tapas de empanadas, vida útil, conservador.
\end{abstract}

\begin{abstract}
$\underline{\text { Abstract }}$
Commonly pie caps at market use polyethylene films as spacers between them. This paper studies the conventional spacers replacement with edible and biodegradable films made with whey protein isolate (WPI) and potassium sorbate as a preservative. Besides facilitating the separation of pie caps, with this application is intended to increase their shelf life. The films made by the compression molding method were used as spacers in pie caps without preservative in their formula (A) and with preservative (B) and they were compared with conventional polyethylene spacers (C). During four months, monthly sensory, microbiological and physicochemical (humidity) evaluations were done on the pie caps, together with humidity and solubility evaluations of the films. None of the samples showed microbiological or sensory deterioration. The sensory attributes showed no or slight difference in study time. Between samples the differences were minor: the best scores were for sample $A$ in color, sample $C$ in flavor, and samples $B$ and $C$ in texture and overall liking. The edible films have an interesting potential for this application, although studies in disguise the flavor of serum should be done.

Keywords: Biodegradable films, pie caps, shelf life, preservative.
\end{abstract}

\section{Introducción}

Por su carácter biodegradable y flexible, las películas comestibles tienen un amplio potencial de uso como alternativa de empaque de alimentos. Éstas son utilizadas en contacto directo con el alimento, cubriendo su superficie externa para separarlo del ambiente exterior o separando superficies internas para aislar la interacción entre sus componentes. En los últimos años se han estudiado una gran diversidad de aplicaciones en películas elaboradas con diferentes matrices alimentarias de polímeros naturales obtenidos de proteínas animales y vegetales, lípidos, polisacáridos, gomas, etcétera, con una extensa variedad en propiedades mecánicas y de barrera (Daraba, 2008).

Existen estudios de aplicación de películas para la mejora de la calidad y vida útil de los alimentos, con el fin de ser utilizadas como empaque para pequeñas porciones, entre otras, peras, frijoles, nueces y frutillas. También se utilizan en las interfases heterogéneas de algunos alimentos como pizzas y tartas para la prevención de su deterioro (Bourtoom et al., 2008). Una de las ventajas que presentan es el control del desarrollo microbiano, en algunos casos formando parte de la matriz, en el quitosano, por ejemplo, y en otros con el agregado de aditivos, alcanzando buenos resultados (Dutta et al., 2009; Kristo et al., 2008; Lungu y Johnson, 2005; McCormick et al., 2005).

Las películas producidas con aislado de proteínas lácteas (WPI) son excelentes candidatos por sus características funcionales y nutricionales, pues presentan buenas propiedades mecánicas y de barrera al oxígeno (Escobar et al., 2008). Adicionalmente, el uso de 
proteínas lácteas para elaboración de películas biodegradables a nivel industrial tendría un beneficioso impacto ambiental, con otro producto para el aprovechamiento del suero de quesería.

Las tapas de empanadas utilizan películas de polietileno como separadores entre una y otra. Presentan dificultad de separación debido a la adherencia de los separadores utilizados, los cuales son, además, un desecho para el medio ambiente.

La sustitución de los separadores de polietileno por las películas de aislado de suero de proteína láctea tiene la ventaja de que las segundas pueden ser comestibles, son biodegradables, facilitan más la separación de las tapas y permiten incluir un conservante en la película, evitando que sea incorporado en la formulación de la masa.

En la actualidad la empresa RH, productora de las empanadas, las comercializa con una vida útil de dos meses.

Este trabajo estudia la vida útil de las tapas de empanadas, utilizando películas comestibles y de polietileno tradicional durante cuatro meses. Se evalúan la calidad microbiológica y sensorial de las tapas de empanadas, su variación de humedad y de actividad de agua. Se estudian, también, los cambios de humedad y la solubilidad de las películas a lo largo de ese tiempo.

\section{Materiales y Métodos}

\section{Preparación de las películas por método de compresión y moldeo}

Las películas se prepararon a partir de una mezcla de WPI (proteína aislada de suero lácteo PROVON ${ }^{\circledR} 290,90 \%$ p/p de Glanbia Nutritionals, WI, USA), glicerol (Merck) y agua. Se elaboraron manteniendo una relación de WPI/plastificante 2,3/1 y un $18 \%(\mathrm{p} / \mathrm{p})$ de humedad. La mezcla se batió en batidora manual a la mínima velocidad durante 15 minutos y se amasó; posteriormente se almacenó en bolsas de polietileno a $5{ }^{\circ} \mathrm{C}$ durante 24 horas para equilibrar sus componentes. Se colocaron esferas de $3 \mathrm{~g}$ de mezcla entre las placas de la prensa de laboratorio (Toyoseiki, P2-25T), se sometieron a temperatura de $140{ }^{\circ} \mathrm{C}$ y presión de $1 \mathrm{MPa}$ durante 2 minutos y se retiraron luego de 3 minutos de enfriamiento. En las películas con sorbato de potasio en una proporción del $10 \%$ éste fue disuelto en el agua de la mezcla antes de agregarse.

\section{Elaboración de tapas de empanadas utilización de las películas}

Según la formulación tradicional de la empresa RH alimentos, se realizaron dos tipos de formulaciones de las tapas de empanadas: con y sin sorbato de potasio en la masa. El peso de cada tapa de empanada fue de $33 \mathrm{~g}$. Las tapas se envasaron en bolsas de polietileno que contenían 10 unidades cada una.

Una vez elaboradas las masas de las tapas empanadas, se colocaron los separadores entre las tapas según los casos de estudio:

A: Tapas de empanadas sin sorbato de potasio en la masa, con película como separador.

B: Tapas de empanadas con sorbato de potasio en la masa, con película como separador.

C: Tapas de empanadas con sorbato de potasio en la masa, con separador de polietileno tradicional.

Luego de colocar los separadores entre las tapas (polietileno o película, dependiendo del caso), fueron envasadas en las bolsas de polietileno que utiliza la empresa habitualmente al comercializar. Las muestras se mantuvieron refrigeradas a $4{ }^{\circ} \mathrm{C}$ durante los cuatro meses de estudio.

Todos los análisis fueron realizados a tiempo cero ( 24 horas luego de elaboradas las películas y/o envasadas las tapas de empanadas) uno, dos, tres y cuatro meses.

\section{Humedad y solubilidad en agua de las películas}

La solubilidad en agua se definió como el porcentaje de materia seca de la película solubilizada después de 24 horas de inmersión en agua (Gontard et al., 1994). El contenido inicial de materia seca (\%) y de humedad se determinó gravimétricamente secando las muestras de las películas a $105{ }^{\circ} \mathrm{C}$ en una estufa con circulación de aire por 24 horas. Se cortaron tres discos de película de $2 \mathrm{~cm}$ de diámetro, se pesaron y se colocaron en un vaso de bohemia con $50 \mathrm{~mL}$ de agua. Se llevaron a una incubadora con agitación (New Brunswick Scientific Co., modelo G25) a $20{ }^{\circ} \mathrm{C}$ y $62 \mathrm{rpm}$. A continuación, las muestras de películas se quitaron de los vasos y se secaron en una estufa a $105^{\circ} \mathrm{C}$ durante 24 horas, para así determinar la materia seca no soluble en agua. Restando este valor del peso de materia seca inicial se obtuvo el peso de materia seca que se solubilizó en agua durante 24 horas y se expresó como un porcentaje de la materia seca inicial. Los ensayos se realizaron por cuadriplicado.

\section{Humedad y actividad de agua de las tapas de empanadas}

Lahumedad se determinó pormétodogravimétrico en estufaa $105^{\circ} \mathrm{C}$ con circulación de aire durante 24 horas. Se determinó actividad de agua con equipo Aqualab Series $3 \mathrm{TE}$ (a $25^{\circ} \mathrm{C}$ ). Los ensayos se realizaron por triplicado.

\section{Evaluación sensorial y vida útil de tapas de empanadas}

Las tapas de empanadas se calentaron en horno a $230{ }^{\circ} \mathrm{C}$ durante 5 minutos y se realizó con consumidores la evaluación sensorial de los atributos color, sabor, textura y agrado general, utilizando una escala hedónica de nueve puntos (1-Me disgusta muchísimo a 9-Me gusta muchísimo), a los 0, 1, 2, 3 y 4 meses.

Las tapas sin hornear fueron evaluadas por un panel de siete jueces, quienes evaluaron el color, el olor y la dificultad de separación de las tapas.

\section{Recuento de hongos y levaduras de las tapas de empanadas}

Se determinó según APHA. Los ensayos se realizaron por triplicado.

\section{Análisis estadístico}

Los resultados fueron evaluados utilizando análisis de varianza (ANOVA), con el software Infostat, y las comparaciones fueron realizadas por test de Tukey. 


\section{Resultados y Discusión}

\section{Humedad y solubilidad de películas. Humedad $y$ actividad de agua en tapas de empanadas}

\begin{tabular}{|c|c|c|c|c|c|c|}
\hline & \multirow{2}{*}{ Muestra } & \multicolumn{5}{|c|}{ Tiempo (meses) } \\
\cline { 3 - 7 } & & $\mathbf{0}$ & $\mathbf{1}$ & $\mathbf{2}$ & $\mathbf{3}$ & $\mathbf{4}$ \\
\hline \multirow{2}{*}{ Humedad (\%) } & $\mathbf{A}$ & $35.8 \mathrm{~b}$ & $42.4 \mathrm{~d}$ & $44.5 \mathrm{de}$ & $45.8 \mathrm{e}$ & $44.4 \mathrm{de}$ \\
\cline { 2 - 7 } & $\mathbf{B}$ & $31.7 \mathrm{a}$ & $38.8 \mathrm{c}$ & $43.0 \mathrm{~d}$ & $42.7 \mathrm{~d}$ & $43.0 \mathrm{~d}$ \\
\hline \multirow{2}{*}{ Solubilidad (\%) } & $\mathbf{A}$ & $27.8 \mathrm{de}$ & $21.2 \mathrm{ab}$ & $22.2 \mathrm{ab}$ & $20.5 \mathrm{a}$ & $21.0 \mathrm{ab}$ \\
\cline { 2 - 7 } & $\mathbf{B}$ & $29.8 \mathrm{e}$ & $26.0 \mathrm{~cd}$ & $22.0 \mathrm{a} \mathrm{b}$ & $23.9 \mathrm{bc}$ & $22.2 \mathrm{ab}$ \\
\hline
\end{tabular}

Tabla 1. Humedad y solubilidad de las películas comestibles A y $\mathrm{B}$ durante los cuatro meses de estudio. Medias con letras iguales no representan diferencias significativas $(p<0,05)$.

Los separadores de tapas de empanadas A y B (sin y con conservador) presentaron un aumento de la humedad de las películas en los dos primeros meses de almacenamiento, y no se registraron diferencias significativas de humedad entre las muestras durante los meses posteriores (Tabla 1). Esta tendencia se observó en los dos casos estudiados; la humedad de las películas utilizadas en las muestras con sorbato de potasio (B) fue menor a las sin sorbato (A) en todo el estudio. La humedad aumentó tras dos meses de almacenamiento, de 35,8 a $44,5 \%$ y de 31,7 a $43,0 \%$ en las muestras A y B, respectivamente.

Contrariamente al aumento de humedad, se produjo una disminución de la solubilidad de las películas, desde 27,8 a 21,2 al mes de almacenado en las películas sin conservador (A) y de 29,8 a 22,0 luego de dos meses de almacenadas en las películas con conservador (B). La disminución de solubilidad al agua de las películas es deseada para la mejora de la estabilidad de la película en contacto con alimentos que presentan humedad, por lo que el almacenamiento le confiere mayor estabilidad desde el punto de vista de solubilidad a la película. Los valores de solubilidad encontrados fueron de $29,8 \%$, los cuales disminuyeron con el tiempo de almacenamiento y fueron menores que los presentados en el estudio realizado por Escobar et al. (2009), de $39 \%$ de solubilidad al agua.

\begin{tabular}{|c|c|c|c|c|c|c|}
\hline \multirow{2}{*}{ Muestra } & \multicolumn{5}{|c|}{ Tiempo (meses) } \\
\cline { 3 - 7 } & & $\mathbf{0}$ & $\mathbf{1}$ & $\mathbf{2}$ & $\mathbf{3}$ & $\mathbf{4}$ \\
\hline \multirow{3}{*}{ Humedad (\%) } & $\mathbf{A}$ & $30.3 \mathrm{abc}$ & $30.9 \mathrm{c}$ & $30.8 \mathrm{c}$ & $30.8 \mathrm{c}$ & $30.7 \mathrm{c}$ \\
\cline { 2 - 7 } & $\mathbf{B}$ & $30.7 \mathrm{c}$ & $30.5 \mathrm{bc}$ & $29.5 \mathrm{ab}$ & $29.9 \mathrm{abc}$ & $29.7 \mathrm{ab}$ \\
\cline { 2 - 7 } & $\mathbf{C}$ & $32.7 \mathrm{~d}$ & $32.7 \mathrm{~d}$ & $31.9 \mathrm{~d}$ & $31.9 \mathrm{~d}$ & $32.1 \mathrm{~d}$ \\
\hline \multirow{3}{*}{$\mathbf{a}_{\mathbf{w}}$} & $\mathbf{A}$ & - & $0.923 \mathrm{a}$ & $0.930 \mathrm{~b}$ & $0.930 \mathrm{~b}$ & $0.931 \mathrm{~b}$ \\
\cline { 2 - 7 } & $\mathbf{B}$ & - & $0.920 \mathrm{a}$ & $0.932 \mathrm{~b}$ & $0.930 \mathrm{~b}$ & $0.927 \mathrm{ab}$ \\
\cline { 2 - 7 } & $\mathbf{C}$ & - & $0.949 \mathrm{c}$ & $0.948 \mathrm{c}$ & $0.942 \mathrm{c}$ & $0.945 \mathrm{c}$ \\
\hline
\end{tabular}

Tabla 2. Humedad y actividad de agua de las tapas de empanadas con los distintos separadores utilizados A, B y C, durante los 4 meses de estudio. Medias con letras iguales no representan diferencias significativas $(p<0,05)$.

Las tapas de empanadas en las que se usó polietileno como separador siempre mantuvieron un nivel de humedad superior al de las tapas de empanadas que usaron películas como separadores. Esto puede deberse a que en las 24 horas de almacenamiento que transcurrieron entre el envasado y la realización del primer análisis (tiempo cero) haya existido una migración de humedad de las tapas hacia las películas comestibles, lo cual no ocurrió con los separadores de polietileno. En estudios reportados por Escobar et al. (2009) de películas de WPI con sorbato de potasio como conservador se encontraron valores de humedad de películas del $19 \%$. La diferencia entre este valor y los hallados en este estudio, de $31,7 \%$, podría también fundamentar la migración de humedad de las tapas hacia las películas, teniendo en cuenta además que la relación en peso de tapa de empanada con la película comestible es de 33:3. Una pequeña diferencia de humedad en la tapa se transforma en una mayor diferencia en la película.

\section{Evaluación sensorial}

\begin{tabular}{|c|c|c|c|c|c|c|}
\hline \multirow{2}{*}{ Atributo } & \multirow{2}{*}{ Muestra } & \multicolumn{5}{|c|}{ Tiempo (meses) } \\
\cline { 2 - 7 } & & $\mathbf{0}$ & $\mathbf{1}$ & $\mathbf{2}$ & $\mathbf{3}$ & $\mathbf{4}$ \\
\hline \multirow{3}{*}{ Sabor } & $\mathbf{A}$ & $5.8 \mathrm{ab}$ & $5.8 \mathrm{ab}$ & $5.7 \mathrm{ab}$ & $5.4 \mathrm{ab}$ & $5.6 \mathrm{ab}$ \\
\cline { 2 - 7 } & $\mathbf{B}$ & $6.1 \mathrm{ab}$ & $5.7 \mathrm{ab}$ & $6.0 \mathrm{ab}$ & $6.6 \mathrm{~b}$ & $4.8 \mathrm{a}$ \\
\cline { 2 - 7 } & $\mathbf{C}$ & $6.4 \mathrm{~b}$ & $6.4 \mathrm{~b}$ & $6.6 \mathrm{~b}$ & $5.5 \mathrm{ab}$ & $6.7 \mathrm{~b}$ \\
\hline \multirow{3}{*}{ Textura } & $\mathbf{A}$ & $5.4 \mathrm{abc}$ & $4.8 \mathrm{ab}$ & $5.4 \mathrm{abc}$ & $5.3 \mathrm{abc}$ & $4.7 \mathrm{a}$ \\
\cline { 2 - 7 } & $\mathbf{B}$ & $5.6 \mathrm{abc}$ & $5.9 \mathrm{abc}$ & $5.8 \mathrm{abc}$ & $6.5 \mathrm{c}$ & $4.8 \mathrm{ab}$ \\
\hline \multirow{3}{*}{ Color } & $\mathbf{C}$ & $5.6 \mathrm{abc}$ & $5.9 \mathrm{abc}$ & $6.2 \mathrm{bc}$ & $5.5 \mathrm{abc}$ & $6.4 \mathrm{c}$ \\
\cline { 2 - 7 } & $\mathbf{A}$ & $6.5 \mathrm{ab}$ & $6.4 \mathrm{ab}$ & $6.3 \mathrm{ab}$ & $6.4 \mathrm{ab}$ & $6.2 \mathrm{ab}$ \\
\cline { 2 - 7 } & $\mathbf{C}$ & $6.8 \mathrm{~b}$ & $6.9 \mathrm{~b}$ & $6.9 \mathrm{~b}$ & $6.9 \mathrm{~b}$ & $6.2 \mathrm{ab}$ \\
\hline \multirow{3}{*}{$\begin{array}{c}\text { Agrado } \\
\text { general }\end{array}$} & $\mathbf{A}$ & $5.6 \mathrm{ab}$ & $5.4 \mathrm{ab}$ & $5.4 \mathrm{ab}$ & $5.5 \mathrm{ab}$ & $5.3 \mathrm{ab}$ \\
\cline { 2 - 7 } & $\mathbf{B}$ & $5.9 \mathrm{ab}$ & $5.8 \mathrm{ab}$ & $5.9 \mathrm{ab}$ & $6.7 \mathrm{~b}$ & $5.0 \mathrm{a}$ \\
\hline & $\mathbf{C}$ & $6.0 \mathrm{ab}$ & $6.6 \mathrm{ab}$ & $6.2 \mathrm{ab}$ & $5.3 \mathrm{ab}$ & $6.6 \mathrm{~b}$ \\
\hline
\end{tabular}

Tabla 3. Resultados de análisis sensorial con panel de consumidores, evaluando sabor, textura, color y agrado general. Escala utilizada del 1 al 9 . Medias con letras iguales no representan diferencias significativas $(p<0,05)$.

En la Tabla 3 se presentan los resultados de evaluación sensorial con el tiempo. Se observa que en todos los casos de estudio los valores son menores que 7 , atribuible a que las tapas son horneadas y se consumen sin relleno, lo cual no lo hace tan atractivo al consumidor.

Se constata que las muestras de tapas de empanadas sin sorbato de potasio con película como separador (muestras A) tuvieron como único atributo variable a lo largo del tiempo la textura, con el puntaje más bajo a los cuatro meses, el cual disminuyó ligeramente de 5.4 a 4.7.

Las tapas de empanadas envasadas de la forma tradicional (muestras C) mantuvieron los valores de los atributos estables durante los cuatro meses de estudio y presentaron en todos los atributos estudiados el mayor valor en el tiempo 4.

En sabor y textura las tres muestras estudiadas no presentaron diferencias significativas hasta los tres meses. La muestra B presentó el menor valor de sabor en el cuarto mes, mientras que en textura el menor valor lo tuvo la muestra $\mathrm{A}$.

Las muestras A y B obtuvieron mayor puntuación en el atributo color que la muestra $\mathrm{C}$ hasta los dos meses del estudio. Esto puede deberse a que en el caso de las muestras en contacto con películas comestibles se haya dado una migración de proteínas de suero a las tapas, lo cual pudo conferir un color más brilloso.

De la evaluación que realizaron los jueces acerca de las tapas sin hornear, todos constataron que las tapas de las muestras A y C tenían un color y olor característico, mientras que solamente un juez halló en la muestra B un color amarillento en la masa. En cuanto al olor, solo dos jueces consignaron una diferencia en las tapas de las muestras A y los demás lo consideraron característico. En relación a la dificultad de separar las tapas de empanadas de los separadores, todos los jueces evaluaron fáciles de separar a las muestras $\mathrm{B}$ y un juez vio dificultad en la separación de la muestra A. Respecto a las muestras 
con separadores de polietileno, cinco de los siete jueces encontraron dificultad en la separación.

\section{Recuento de hongos y levaduras}

En la Tabla 4 se expresan los valores microbiológicos estudiados, donde se observa que no hubo variación del recuento de hongos y levaduras durante los cuatro meses de estudio.

\begin{tabular}{|c|c|c|c|c|c|c|}
\hline \multirow{2}{*}{ Muestra } & & \multicolumn{5}{|c|}{ Tiempo (meses) } \\
\cline { 3 - 7 } & & $\mathbf{0}$ & $\mathbf{1}$ & $\mathbf{2}$ & $\mathbf{3}$ & $\mathbf{4}$ \\
\hline \multirow{3}{*}{$\mathbf{A}$} & $\mathbf{A 1}$ & $<10(\cdot)$ & $20(\cdot)$ & $<10(\cdot)$ & $<10(\cdot)$ & $<10(\cdot)$ \\
\cline { 2 - 7 } & $\mathbf{A 2}$ & $<10(\cdot)$ & $<10(\cdot)$ & $<10(\cdot)$ & $<10(\cdot)$ & $<10(\cdot)$ \\
\cline { 2 - 7 } & $\mathbf{A 3}$ & $<10(\cdot)$ & $<10(\cdot)$ & $<10(\cdot)$ & $<10(\cdot)$ & $<10(\cdot)$ \\
\hline \multirow{3}{*}{ B } & $\mathbf{B 1}$ & $<10(\cdot)$ & $<10(\cdot)$ & $<10(\cdot)$ & $<10(\cdot)$ & $<10(\cdot)$ \\
\cline { 2 - 7 } & $\mathbf{B} 2$ & $<10(\cdot)$ & $<10(\cdot)$ & 20 & $<10(\cdot)$ & $<10(\cdot)$ \\
\cline { 2 - 7 } & $\mathbf{B 3}$ & $2100(\cdot)$ & $<10(\cdot)$ & $<10(\cdot)$ & $<10(\cdot)$ & $<10(\cdot)$ \\
\hline \multirow{3}{*}{$\mathbf{C}$} & $\mathbf{C 1}$ & $<10(\cdot)$ & $<10(\cdot)$ & $<10(\cdot)$ & $<10(\cdot)$ & $<10(\cdot)$ \\
\cline { 2 - 7 } & $\mathbf{C 2}$ & $<10(\cdot)$ & $<10(\cdot)$ & $<10(\cdot)$ & $<10(\cdot)$ & $<10(\cdot)$ \\
\cline { 2 - 7 } & $\mathbf{C 3}$ & $<10(\cdot)$ & $<10(\cdot)$ & $<10(\cdot)$ & $<10(\cdot)$ & $<10(\cdot)$ \\
\hline
\end{tabular}

Tabla 4. Valores de recuento de hongos y levaduras en UFC/g. Se muestran los triplicados de cada tipo de muestra.

(·): Límite de detección de la técnica.

(.•): El recuento corresponde a UFC de levaduras. No se detectaron hongos filamentosos.

\section{Conclusiones}

El estudio fue diseñado inicialmente hasta los cuatro meses de almacenamiento a $4{ }^{\circ} \mathrm{C}$ para superar el tiempo de vida útil que manejaba la empresa, de dos meses. Sin embargo, no se observó un deterioro del producto que permita afirmar que la vida útil hubiera concluido.

Ninguna de las muestras presentó deterioro microbiológico ni sensorial en los cuatro meses estudiados y los atributos presentaron ninguna o ligera diferencia en el tiempo de estudio. Entre muestras las diferencias fueron ligeras; las mejores puntuaciones las tuvieron en color las muestras A y B, en sabor, la muestra C, y en textura y agrado general, las B y C. La utilización de películas realizadas por compresión como método de elaboración permitió que las películas no se solubilizaran en todo el tiempo de estudio debido a los bajos valores de solubilidad presentados. Las películas comestibles tienen un interesante potencial para esta aplicación, aunque se debe trabajar en enmascarar el flavor del suero.

\section{Referencias}

\section{- AMERICAN PUBLIC HEALTH ASSOCIATION. Compendium} of methods for the microbiological examinations of foods. Capítulo 20, 4ta ed. Washington: APHA, 2001.

- BOURTOOM, T. Edible films and coatings: characteristics and properties. En: International Food Research Journal. 2008, 15(3).

- DARABA, A. Future trends in packing: edible, biodegradable coats and films. En: Journal of Environmental Protection and Ecology. 2008, 9(32):654-664.

- DUTTA, P.K.; TRIPATHI, S.; MEHROTRA, G.K.; y DUTTA,
J. Perspectives for chitosan based antimicrobial films in food applications. En: Food Chemistry. 2009, 114:1173-1182.

- ESCOBAR, D.; MÁRQUEZ, R.; REPISO, L.; SALA, A.; SILVERA, C. Elaboración, caracterización y comparación de películas comestibles en base a aislado de proteínas de suero lácteo (WPI). En: INNOTEC. 2008, 3:57-62.

- ESCOBAR, D.; MÁRQUEZ, R.; REPISO, L.; SALA, A., SILVERA, C. Películas biodegradables y comestibles desarrolladas en base a aislado de proteínas de suero lácteo: estudio de dos métodos de elaboración y del uso de sorbato de potasio como conservador. En: INNOTEC. 2009, 4:33-36.

- GONTARD, N.; DUCHEZ, C.; CUQ, J.L.; GUILBERT, S. Edible composite _ lms of wheat gluten and lipids: water vapour permeability and other physical properties. En: International Journal of Food Science and Technology. 1994, (29):39-50.

- KRISTO, E.; KOUTSOUMAINS, K.P.; BILIADERIS, C.G. Thermal, mechanical and water vapor barrier properties of sodium caseinate films containing antimicrobials and their inhibitory action on Listeria monocytogenes. En: Food Hydrocolloids. 2008, 22(3):373-386

- LUNGU, B.; JOHNSON, M.G. Fate of Listeria monocytogenes inoculated onto the surface of model turkey frankfurter pieces treated with zein coatings containing nisin, sodium diacetate, and sodium lactate at $4^{\circ} \mathrm{C}$. En: Journal of Food Protection. 2005, 68:855-859.

- McCORMICK, K.E.; HAN, I.Y.; SHELDON, B.W.; DAWSON, P.L. In-package pasteurization combined with biocideimpregnated films to inhibit Listeria monocytogenes and Salmonella Typhimurium in turkey bologna. En: Journal of Food Science. 2005, 70:53-57. 